\title{
Flow bifurcation routes to chaos of thermocapillary convection for low Prandtl number fluid in shallow annular pool with surface heat dissipation
}

\author{
Li Zhang ${ }^{\mathrm{a}, \mathrm{b}}$, You-Rong $\mathrm{Li}^{\mathrm{a}, *}$, Chun-Mei Wu ${ }^{\mathrm{a}}$, Qiu-Sheng Liu ${ }^{\mathrm{c}}$

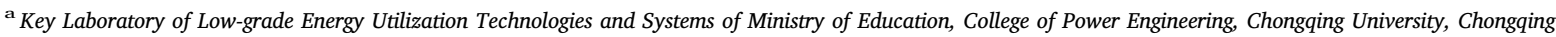 \\ 400044, China \\ ${ }^{\mathbf{b}}$ Chongqing Nanfang Translators College of Sichuan International Studies University, Chongqing 401120, China \\ ${ }^{\mathrm{c}}$ Key Laboratory of Microgravity (National Microgravity Laboratory), Institute of Mechanics, Chinese Academy of Sciences, Beijing, 100190, China
}

\section{A R T I C L E I N F O}

\section{Keywords:}

Flow bifurcation

Thermocapillary convection

Low Prandtl number

Shallow annular pool

Surface heat dissipation

\begin{abstract}
A B S T R A C T
In order to understand clearly the transition characteristics of thermocapillary convection of low Prandtl number fluid in shallow annular pool with surface heat dissipation, some bifurcation routes to chaos of thermocapillary convection have been numerically investigated by using the finite volume method in this paper. The annular pool was filled with the low Prandtl (Pr) number fluid of $P r=0.011$. The range of Biot number is from 0 to 1 . Results indicate that the flow bifurcation route of thermocapillary convection depends intensively on surface heat dissipation. When surface heat dissipation is smaller, with the increase of Marangoni number the flow bifurcation sequence of thermocapillary convection is two-dimensional axisymmetric steady flow $\rightarrow$ three-dimensional steady flow $\rightarrow$ coexisting hydrothermal waves and radial moving waves $\rightarrow$ radial moving waves $\rightarrow$ chaos. When surface heat dissipation is larger, the bifurcation sequence becomes simple as two-dimensional axisymmetric steady flow $\rightarrow$ hydrothermal waves $\rightarrow$ chaos. Furthermore, every bifurcation of thermocapillary convection is always accompanied by the variations of the wave number and the oscillatory frequency. Furthermore, the temperature fluctuation amplitude on the free surface increases gradually with the increase of Marangoni number.
\end{abstract}

\section{Introduction}

Thermocapillary convection driven by surface tension gradient on the free surface has been widely studied and many fruitful results with an adiabatic free surface have been achieved [1-4]. Thermocapillary convection instability is a fundamental phenomenon encountered in a vast variety of situations, ranging from the biomedical science to the engineering system. With the increase of the applied temperature gradient, thermocapillary convection will bifurcate from steady flow to various oscillatory flows, and finally to chaotic flow or turbulence. The intriguing behavior exhibited by these travelling perturbations in various oscillatory flows has been received considerable attention. As early as 1980, Gollub and Benson [5] has observed four transition pathways on Rayleigh-Bénard convection from laminar flow to turbulent flow by the experimental method of Laser Doppler velocimetry. Smith and Davis $[6,7]$ showed that thermocapillary convection in laterally-heated planar layers is naturally vulnerable to the formation of an oscillatory flow pattern characterized by a myriad of thermal wave-like patterns propagating along the gas-liquid interface, i.e. hydrothermal waves (HTWs). Meanwhile, Smith [8] further explained the physical mechanism of two types of thermal convection instability. Villers and Platten [9] investigated buoyancy-thermocapillary convection in acetone solution by experiments and numerical simulations. The results showed that, with the increase of Marangoni number, the convection will experience three states: single vortex steady flow, multi vortex steady flow and unsteady flow. Edoardo et al. [10] developed a numerical analysis of buoyancy-driven natural convection of a fluid in a three-dimensional shallow cavity with a horizontal temperature gradient along the larger dimension for the low Prandtl number $(P r=0.015)$, and the Ruelle-Takens bifurcation sequence has been observed as the transition mechanism to chaos. Zhu et al. [11] presented the experiments in a rectangular pool with the applied temperature difference between the two sidewalls adjusted in the range of $(0-43){ }^{\circ} \mathrm{C}$ and observed various dynamic states. They discussed the relationship between oscillatory frequency and Marangoni number. Li et al. [12-15] simulated numerically thermocapillary convection for the low $(P r=0.011)$ and moderate $(P r=6.7)$ Prandtl number fluids in the annular liquid pool and analyzed the physical mechanism of the hydrothermal wave formation. Gillon and Homsy [16] studied buoyancythermocapillary convection of $0.65 \mathrm{cSt}$ silicone oil layer in a rectangular

\footnotetext{
* Corresponding author.

E-mail address: liyourong@cqu.edu.cn (Y.-R. Li).
} 


$\begin{array}{ll}\text { Nomenclature } \\ A & \text { dimensionless temperature amplitude } \\ B i & \text { Biot number } \\ c_{\mathrm{p}} & \text { specific heat capacity, } \mathrm{kJ} /(\mathrm{kg} \cdot \mathrm{K}) \\ d & \text { depth of annular pool, } \mathrm{m} \\ F & \text { dimensionless frequency } \\ h & \text { convective heat transfer coefficient, } \mathrm{W} /\left(\mathrm{m}^{2} \cdot \mathrm{K}\right) \\ m & \text { wave number } \\ M a & \text { Marangoni number } \\ p & \text { pressure, Pa } \\ P & \text { dimensionless pressure } \\ P r & \text { Prandtl number } \\ r & \text { radius, m } \\ R & \text { dimensionless radius } \\ t & \text { time, } \mathrm{s} \\ T & \text { temperature, } \mathrm{K} \\ u & \text { radial velocity, } \mathrm{m} / \mathrm{s} \\ U & \text { dimensionless radial velocity } \\ v & \text { azimuthal velocity, } \mathrm{m} / \mathrm{s} \\ V & \text { dimensionless azimuthal velocity } \\ V & \text { dimensionless velocity vector } \\ w & \text { axial velocity, } \mathrm{m} / \mathrm{s}\end{array}$

W

$z$

Z

Greek symbols

$\alpha \quad$ thermal diffusivity, $\mathrm{m}^{2} / \mathrm{s}$

$\varepsilon \quad$ aspect ratio

$\gamma_{\mathrm{T}} \quad$ temperature coefficient of surface tension, $\mathrm{N} /(\mathrm{m} \cdot \mathrm{K})$

$\eta \quad$ radius ratio

$\mu \quad$ dynamic viscosity, $\mathrm{kg} /(\mathrm{m} \cdot \mathrm{s})$

$\nu \quad$ kinematic viscosity, $\mathrm{m}^{2} / \mathrm{s}$

$\Theta \quad$ dimensionless temperature

$\rho \quad$ density, $\mathrm{kg} / \mathrm{m}^{3}$

$\tau \quad$ dimensionless time

$\psi \quad$ dimensionless stream function

\section{Subscripts}

$\begin{array}{ll}0 & \text { ambient } \\ \text { i } & \text { inner } \\ \max & \text { maximum } \\ \text { o } & \text { outer }\end{array}$

pool by particle image velocimetry (PIV) and explained the effect of Marangoni number on the flow pattern transition. Furthermore, some scholars have observed the stationary longitudinal roll pattern and the HTWs in an annular pool by experiments [17-21].

It is not hard to see that the previous researches are based on the adiabatic free surface and ignore surface heat dissipation. In fact, owing to the interfacial non-equilibrium effect, heat dissipation to the environment on the free surface becomes inevitable, which greatly influences the temperature distribution along the free surface and then changes the flow bifurcation routes of thermocapillary convection, especially for low Prandtl number fluids. Until now, thermocapillary convection with surface heat dissipation in an annular pool subjected to a horizontal temperature gradient is few investigated. Jing et al. [22] carried out three-dimensional (3-D) numerical simulations of LiNbO3 melt flow in an open crucible that the sidewall is heated at constant heat flux, the bottom is adiabatic and the radiation heat loss from the melt surface to the ambient is taken into account. They analyzed the mechanism of the well-known surface spoke patterns, and certified that the spoke patterns are caused by Marangoni instability in the thin thermal boundary layer near the free surface. Zhang et al. [23] studied vaporing thermal convection on the thin liquid layer in the rectangular cavity by the systematic experimental observations. The results showed thermal convection will occur in the fluid layer as long as surface evaporation as the driving force is strong enough whether the bottom of the liquid layer is heated or cooled. Li et al. [24] investigated thermocapillary-buoyancy convection of $0.65 \mathrm{cSt}$ silicone oil in a rectangular tank and drew the conclusion that with the increase of Marangoni number, the flow becomes unsteady flow; with the decrease of relative concentration in the air, the critical Marangoni number of flow transition increases. Meanwhile, the linear stability analysis shows that as the interfacial evaporation increases, the flow instability will be suppressed. It can be seen that thermocapillary convection with the evaporating interface is much more complicated than that with an adiabatic free surface. Liu et al. [25-27] made experimental observations and numerical simulations on thermocapillary convection of the evaporating liquid layer in the rectangular pool subjected to a horizontal temperature gradient. The results showed that the interfacial evaporation has a great influence on the instability of thermocapillary convection, and the evaporation intensity is related to the non-equilibrium degree through the evaporation interface, which is dependent on the evaporation Biot $(\mathrm{Bi})$ number on the free surface. Hoyas et al. [28-30] studied the instabilities appearing in a cylindrical annulus with the heating bottom and the opening free surface to the atmosphere by linear stability analysis. After the flow destabilizes, the appearance of the various flow patterns and the flow bifurcation routes to chaos are mainly dependent on Marangoni number, Biot number and Prandtl number. Doumenc et al. [31] and Touazi et al. [32] performed a linear stability analysis on the transient Rayleigh-Bénard-Marangoni convective instability due to surface cooling induced by solvent evaporation and determined the critical Marangoni and Rayleigh numbers when both thermocapillary force and buoyancy driving force are taken into account.

The research on the mechanism of thermocapillary convection instability is beneficial to explore flow bifurcation routes. When surface heat dissipation is taken into account, to identify the flow bifurcation routes to chaos of thermocapillary convection for low Prandtl number fluid in shallow annular pool, this paper presented a series of 3-D numerical simulations.

\section{Problem statement}

\subsection{Physical model}

A sketch of the problem is provided in Fig. 1. A shallow annular pool with inner radius $r_{\mathrm{i}}$, outer radius $r_{\mathrm{o}}$ and depth $d$ is filled with the low Prandtl number fluid. The radius ratio and the aspect ratio of the

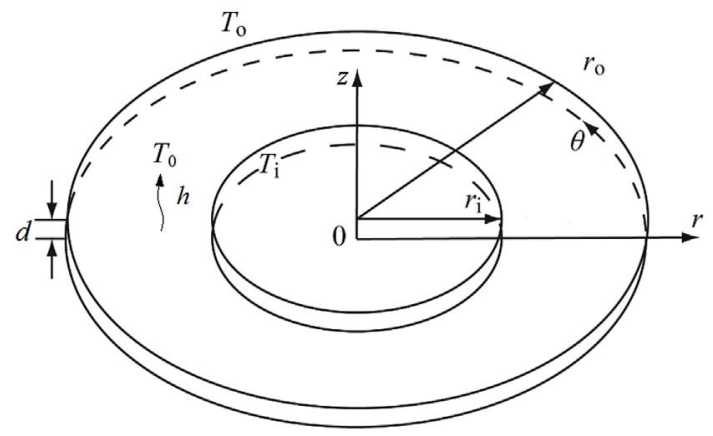

Fig. 1. Physical model and the coordinate system. 
annular pool are defined as $\eta=r_{\mathrm{i}} / r_{\mathrm{o}}$ and $\varepsilon=d /\left(r_{\mathrm{o}}-r_{\mathrm{i}}\right)$, respectively. The bottom is considered to be thermally adiabatic, which satisfies noslip boundary conditions. The free surface is flat and non-deformable, which has heat dissipation to ambient with the temperature $T_{0}$. The total surface heat transfer coefficient is $h$. The inner and outer cylinders are respectively kept at specific temperatures $T_{\mathrm{i}}$ and $T_{\mathrm{o}}\left(T_{\mathrm{o}}>T_{\mathrm{i}}\right)$.

For simplicity, the following assumptions are considered. (1) The fluid is assumed to be an incompressible Newtonian fluid. (2) A constant physical property assumption is applicable except for the surface tension. (3) The velocity is small and the flow is laminar. (4) The free surface is flat and non-deformable. (5) If there is evaporation cooling on the free surface, the evaporation rate is too slow to affect the depth of the pool.

\subsection{Mathematical formulation}

By applying $\left(r_{\mathrm{o}}-r_{\mathrm{i}}\right), \nu /\left(r_{\mathrm{o}}-r_{\mathrm{i}}\right),\left(r_{\mathrm{o}}-r_{\mathrm{i}}\right)^{2} / \nu$ and $\mu \nu /\left(r_{\mathrm{o}}-r_{\mathrm{i}}\right)^{2}$ as scale quantities for length, velocity, time and pressure, we have

$$
\begin{aligned}
(R, Z) & =\frac{(r, z)}{r_{o}-r_{i}},(U, V, W)=\frac{(u, v, w)}{\nu /\left(r_{o}-r_{i}\right)}, \tau=\frac{t}{\left(r_{o}-r_{i}\right)^{2} / \nu}, P \\
& =\frac{p}{\mu \nu /\left(r_{o}-r_{i}\right)^{2}}
\end{aligned}
$$

Based on the above assumptions, the dimensionless flow and heat transfer equations together with the proper boundary conditions can be expressed by the following forms:

$\nabla \cdot \boldsymbol{V}=0$

$\frac{\partial \boldsymbol{V}}{\partial \tau}+\boldsymbol{V} \cdot \nabla \boldsymbol{V}=-\nabla P+\nabla^{2} \boldsymbol{V}$

$\frac{\partial \Theta}{\partial \tau}+\boldsymbol{V} \cdot \nabla \Theta=\frac{1}{P r} \nabla^{2} \Theta$

In equations, $\boldsymbol{V}, \tau$ and $P$ are the dimensionless velocity vector, time and pressure, respectively. $\Theta$ is the dimensionless temperature, which is defined as $\Theta=\left(T-T_{\mathrm{i}}\right) /\left(T_{\mathrm{o}}-T_{\mathrm{i}}\right)$.

The initial conditions are:

$\tau=0, U=V=W=0, \Theta=-\ln [R(1-\eta) / \eta] / \ln \eta$

The following boundary conditions are considered:

At the inner cylinder $\left(R=R_{\mathrm{i}}=r_{\mathrm{i}} /\left(r_{\mathrm{o}}-r_{\mathrm{i}}\right)=\eta /(1-\eta), 0 \leq Z \leq \varepsilon\right)$ : $U=V=W=0, \Theta=0$;

At the outer cylinder $\left(R=R_{\mathrm{o}}=r_{\mathrm{o}} /\left(r_{\mathrm{o}}-r_{\mathrm{i}}\right)=1 /(1-\eta), 0 \leq Z \leq \varepsilon\right)$ : $U=V=W=0, \Theta=1$;

At the bottom $(Z=0, \eta /(1-\eta)<R<1 /(1-\eta))$ :

$U=V=W=0, \partial \Theta / \partial Z=0$.

At the free surface $(Z=\varepsilon, \eta /(1-\eta)<R<1 /(1-\eta))$, the radial velocity $U$ and the azimuthal velocity $V$ depend on the balance effect between the thermocapillary force and the shear stress of the fluid. At the same time, the axial velocity caused by surface evaporation is too small to be neglected. Therefore, $\frac{\partial U}{\partial Z}=-\frac{M a}{\operatorname{Pr}} \frac{\partial \Theta}{\partial R}, \frac{\partial V}{\partial Z}=-\frac{M a}{\operatorname{Pr}} \frac{\partial \Theta}{R \partial \theta}, W=0$

where, $M a$ is Marangoni number, $M a=\gamma_{\mathrm{T}} \Delta T\left(r_{\mathrm{o}}-r_{\mathrm{i}}\right) /(\mu \alpha), \gamma_{\mathrm{T}}$ surface tension temperature coefficient, $\mu$ dynamic viscosity, $\alpha$ thermal diffusivity.

When the total heat transfer coefficient on the free surface is $h$, the thermal boundary condition can be expressed as

$-\lambda \partial T / \partial z=h\left(T-T_{0}\right)$.

In Eq. (9), $\lambda$ is thermal conductivity. Supposing $T_{0}=T_{\mathrm{i}}$, the above formula is also expressed in the dimensionless form

$-\partial \Theta / \partial Z=B i \Theta$

in which, $B i$ is surface heat dissipation Biot number, $B i=h\left(r_{\mathrm{o}}-r_{\mathrm{i}}\right) / \lambda$.

The low Prandtl fluid chosen in this paper is silicon melt with $\operatorname{Pr}=0.011$. In this case, the total surface heat dissipation coefficient is smaller. Therefore, Biot number was limited in the range $0 \leq B i=1.0$. Radius ratio and aspect ratio of an annular pool are respectively fixed at $\eta=0.5$ and $\varepsilon=0.05$.

\subsection{Numerical procedure and validation}

The fundamental equations are discretized by the finite volume method. The central difference approximation is introduced for the diffusion terms while the convective terms are treated by the QUICK scheme. The SIMPLE algorithm is used for correcting simultaneously the pressure and the velocity. The dimensionless time step is in the range between $2 \times 10^{-6}$ and $2 \times 10^{-5}$. In the iterative solution process, when the maximum relative error of temperature and velocity is not greater than $10^{-5}$, the solution is considered to be convergent.

Non-uniform staggered grids of $60^{\mathrm{R}} \times 22^{\mathrm{Z}} \times 240^{\theta}$ which are encrypted near the solid walls and the free surface are applied. To verify the convergence of the mesh, typical simulation results at four different girds are listed in Table 1 at two sets of different Marangoni numbers and Biot numbers. When $M a=5000$ and $B i=1.0$, the flow is steady for the first set of grid while it is 3-D oscillatory for the latter three groups of grids. On the free surface, the oscillating wave number $m$ is essentially the same. In this paper, the wave number is determined by observing the snapshot of surface temperature fluctuation in post-processing after the converged laminar flow solution is obtained. When $M a=8000$ and $B i=0.25$, the flow pattern is the hydrothermal wave for the first set of grid while it is a radial moving wave for the latter three groups of grids. Furthermore, the difference of dimensionless oscillatory fundamental frequency $F_{0}$ between the latter three groups of grids is not more than $3 \%$. Therefore, the selected grid $60^{\mathrm{R}} \times 22^{\mathrm{Z}} \times 240^{\theta}$ is reasonable.

To validate the current numerical scheme, we reproduced numerically thermocapillary-buoyancy convection of silicon melt and successfully obtained the flow patterns observed experimentally by Azami et al. [33] at the same conditions. Then, when the evaporation on the free surface was taken into account, a series of numerical simulations on thermocapillary convection of the fluid with $P r=12$ are carefully carried out at the same conditions as the work of Liu et al. [34]. The obtained free surface temperature profiles are identical with the

Table 1

\begin{tabular}{|c|c|c|c|c|}
\hline \multicolumn{5}{|c|}{$M a=5000, B i=1$} \\
\hline mesh & $40^{\mathrm{R}} \times 22^{\mathrm{Z}} \times 120^{\theta}$ & $50^{\mathrm{R}} \times 22^{\mathrm{Z}} \times 180^{\theta}$ & $60^{\mathrm{R}} \times 22^{\mathrm{Z}} \times 240^{\theta}$ & $60^{\mathrm{R}} \times 30^{\mathrm{Z}} \times 240^{\theta}$ \\
\hline$m$ & 60 & 24 & 25 & 25 \\
\hline$F_{0}$ & Steady flow & 1656 & 1662 & 1655 \\
\hline $\begin{array}{l}M a= \\
\text { mesh }\end{array}$ & $40^{\mathrm{R}} \times 22^{\mathrm{Z}} \times 120^{\theta}$ & $50^{\mathrm{R}} \times 22^{\mathrm{Z}} \times 180^{\theta}$ & $60^{\mathrm{R}} \times 22^{\mathrm{Z}} \times 240^{\theta}$ & $60^{\mathrm{R}} \times 30^{\mathrm{Z}} \times 240^{\theta}$ \\
\hline$m$ & 11 & 15 & 16 & 16 \\
\hline$F_{0}$ & 1246 & 1596 & 1658 & 1656 \\
\hline
\end{tabular}

Mesh convergence check. 
literature results, as shown in Ref. [35]. On the other hand, the threedimensional numerical simulation program used in this paper has also been successfully used to the calculation of thermocapillary convection under various conditions [35-37]. These validations provide enough confidence to the accuracy of the numerical scheme.

\section{Results and discussion}

\subsection{Basic flow}

When the temperature gradient exists on the free surface, the surface tension gradient will drive the fluid flowing from the high temperature wall to the low temperature wall. If the temperature difference between the inner and outer walls is smaller, the flow is two-dimensional (2-D) axisymmetric steady-state flow, which can be described by the streamlines in the meridian plane. The dimensionless flow function is defined as

$U=\frac{1}{R} \frac{\partial \psi}{\partial Z}, W=-\frac{1}{R} \frac{\partial \psi}{\partial R}$.

Fig. 2 revealed the isotherm and streamline distributions with different Biot numbers at $M a=3750$. When Biot number is smaller, the radial temperature gradient near the inner wall is larger, the flow is stronger, and the streamlines are denser. With the increase of Biot number, the radial temperature gradient near the inner wall on the free surface gradually decreases, and the thermocapillary effect weakens. However, near the outer wall, the results are just the opposite. The radial temperature gradient increases and the thermocapillary effect enhances, which make all the thermocapillary flow cell move outward. Furthermore, the isotherms near the outer wall become dense. Meanwhile, the flow strengthens continuously, and the dimensionless flow function increases.

In Fig. 3, the radial distributions of the dimensionless radial and axial temperature gradients on the free surface under different Biot numbers are given. Obviously, when the free surface is adiabatic, the axial dimensionless temperature gradient on the surface is zero. However, when surface heat dissipation is taken into account, a negative

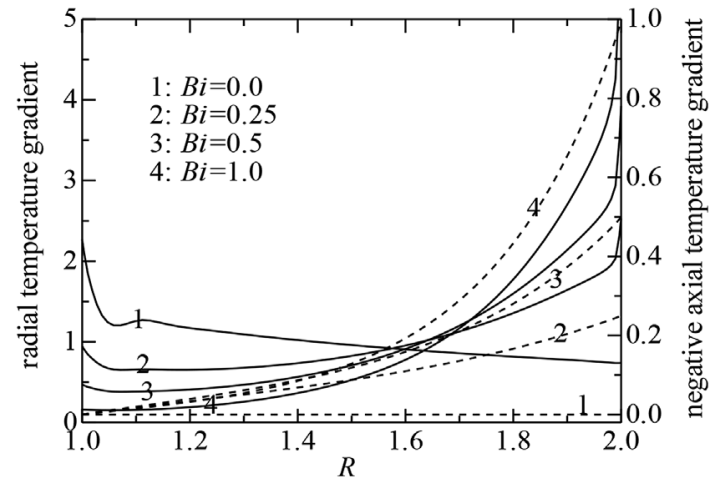

Fig. 3. Variations of the radial (solid lines) and axial (dotted lines) temperature gradients on the free surface at $M a=3750$.

axial temperature gradient is generated near the free surface, which increases monotonically with the increase of Biot number. On the other hand, the axial temperature gradient increases gradually along the radial direction. However, the variation of radial temperature gradient with Biot number is quite complex. Near the inner wall, the radial temperature gradient decreases gradually with the increase of Biot number, while near the outer wall, it increases gradually. With the increase of Marangoni number, this relative variation of the radial temperature gradient and the axial temperature gradient on the free surface will lead to the different flow patterns after the flow destabilizes.

\subsection{Flow bifurcation at a small Biot number}

With the increase of Marangoni number, the flow is strengthened. When Marangoni number exceeds a critical value, three-dimensional disturbances are incubated and their amplitudes increase gradually with time. Finally, different 3-D flow patterns are formed depending on the thermal boundary condition on the free surface. When the surface is adiabatic, the axisymmetric steady flow will bifurcate to the HTWs moving along the azimuthal direction, as reported in Refs. [33,36,39].

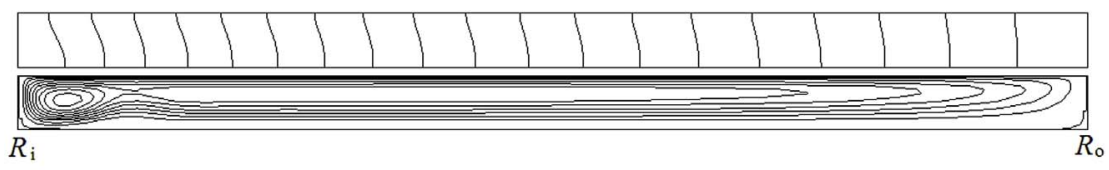

(a) $B i=0, \psi_{\max }=57.70$

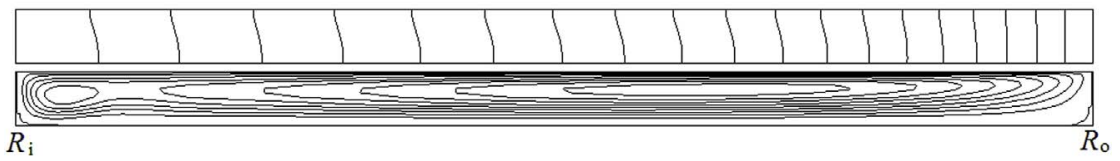

(b) $B i=0.25, \psi_{\max }=58.09$

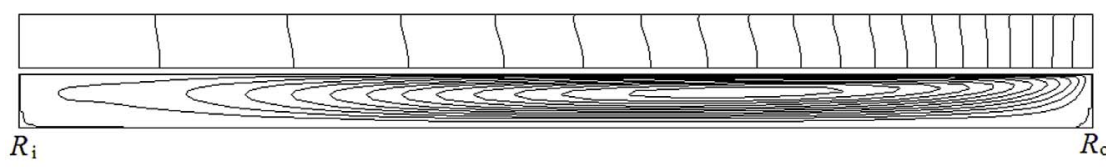

(c) $B i=0.5, \psi_{\max }=67.19$

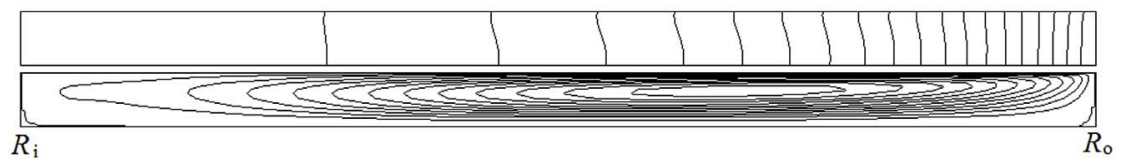

(d) $B i=1.0, \psi_{\max }=76.16$
Fig. 2. Isotherms (up) and streamlines (down) of basic flow in a meridional plane at $M a=3750 . \delta \Theta=0.05, \delta \psi=5$. 
However, when there is heat dissipation on the free surface, at a small Biot number such as $0.05<B i<0.375$, the axisymmetric steady flow after its destabilization firstly evolves into the three-dimensional steady flow (3DSF), which shows a group of radial roll cells arranged in the azimuthal direction [38]. The direction of the rolling cell central axis is the same as the radial direction.

In order to analyze the characteristics of the 3-D flow, the temperature fluctuation $\delta \Theta$ on the free surface is introduced as

$\delta \Theta=\Theta(R, \varepsilon, \theta, \tau)-\frac{1}{2 \pi} \int_{0}^{2 \pi} \Theta(R, \varepsilon, \theta, \tau) \mathrm{d} \theta$

Fig. 4 shows the temperature fluctuation nephogram of 3-D steady flow on the free surface, and the spatial-time diagram (STD) of surface temperature along the circumferential direction at $R=1.16$ with time when $B i=0.125$ and 0.25 respectively. Obviously, at small Biot numbers, the radial temperature gradient on the free surface near the inner wall is larger, and the radial velocity and the velocity gradients are the largest. The axisymmetric steady flow destabilizes near the inner wall at first, so the temperature fluctuation amplitude is the largest. With the increase of Biot number, the radial temperature gradient near the inner wall decreases, which will lead to the decrease of the temperature fluctuation amplitude. For example, when Biot number increases from 0.125 to 0.25 , the maximum temperature fluctuation decreases almost half, but the wave number changes little, only increasing from 38 to 39 .

When Marangoni number continues to increase, the 3DSF will destabilize and evolve into the 3-D unsteady flow. When the free surface is adiabatic, the flow pattern after the flow destabilizes is the HTWs $[35,36,39]$. However, when there exists surface heat dissipation, if Biot number is smaller, such as $B i=0.25$, a set of radial moving waves (RMWs) propagating along the radial direction can be observed on the free surface after the flow destabilizes. It originates near the inner wall and propagates outward along the radial direction [38]. However, the fluctuations of temperature and velocity are not synchronous along the azimuthal direction. At the same time, in the local region, these fluctuations will rotate along the azimuthal direction, as shown in Fig. 5 (a). Therefore, the STD shows the alternation of the high temperature and the low temperature, and there are the partial slant lines. This flow pattern can be considered as a combination of radial moving waves and HTWs (RMWs + HTWs). The fluctuation spectrum of the radial velocity at a monitoring point of $R=1.5$ on the free surface is shown in Fig. 6 (a). Apparently, although the radial moving wave and the hydrothermal wave coexist on the free surface, the fluctuation regularity is very clear, and there is only one dimensionless fluctuation frequency of $F_{0}=1554$ because of the modulation of radial moving waves and hydrothermal waves each other. When Marangoni number increases from 5000 to 6000 , although a combination of the RMWs and HTWs on the free surface is observed, the HTWs moves along both clockwise and counterclockwise directions, as shown in Fig. 5 (b). Simultaneously, the fundamental fluctuation frequency decreases slightly to $F_{0}=1531$. As the flow strengthens, the fluctuation becomes more complex and a harmonic frequency of $F_{1}=3196 \approx 2 F_{0}$ appears on the spectrogram, as shown in Fig. 6(b).

As Marangoni number continues to increase, the flow is further enhanced, and the temperature and velocity fluctuations near the inner wall move outward with the radial backflow near the bottom. Fig. 5(c) shows an instantaneous surface temperature fluctuation at $M a=8000$. The hydrothermal waves apparently disappeared, and there is only the RMWs on the free surface. Therefore, the STD diagram presents the alternation of high temperature and low temperature, just like an international chessboard. Fig. 7 presents temperature fluctuation and streamlines of radial moving wave flow pattern during a period on a meridional plane at $M a=8000$. As can be seen from the diagram, there is always a strong secondary flow cell near the inner wall, which will continuously strengthen and attenuate over time, thus causing oscillations of velocity and temperature. The oscillation propagates toward the outward wall along the radial direction with the backflow. Since the temperature oscillations in the liquid pool are caused by secondary flow cells near the inner wall, the maximum temperature fluctuation does not occur at the free surface, but in the liquid pool. Similarly, because the flow is enhanced, the fluctuation amplitudes of temperature and velocity increase, and the fluctuation regular pattern becomes more complex. Besides the existence of a fundamental fluctuation frequency of $F_{0}=1665$, there are many harmonic frequencies, such as $F_{1}=3329 \approx 2 F_{0}$ and $F_{2}=4957 \approx(3 / 2) F_{1} \approx 3 F_{0}$, as shown in Fig. 6(c).

When Marangoni number exceeds about 10000, the flow becomes very strong, and the temperature fluctuations become disorganized and irregular, as shown in Fig. 5(d). The radial velocity fluctuation spectrum at a monitoring point on the free surface of $R=1.5$ shows that the flow has evolved into chaotic flow (CF), as shown in Fig. 6 (d). Obviously, in this case, 3DSF transits to chaotic flow by the period-halving bifurcation with the increase of Marangoni number.

\subsection{Flow bifurcation at a large Biot number}

When surface heat dissipation is larger, such as $0.375 \leq B i=1.0$, the flow near the inner wall is very weak, as shown in Fig. 2(c) and (d). As a result, with the increase of Marangoni number, 2-D axisymmetric steady flow will not bifurcate 3-D steady flow after the flow destabilization, and directly evolves into the HTWs propagating along the azimuthal direction, instead. The HTWs can propagate along the clockwise (C. W.) or the counterclockwise (C. C. W.), as shown in Fig. 8(a) and (b). At the same time, compare Fig. 8 (a), (b) and (c), it can be seen that, with the increase of Biot number, the flow near the inner wall weakens, and the velocity and temperature fluctuation amplitudes decrease. Therefore, the HTWs gradually move to the outer wall, the maximum temperature fluctuation on the free surface is also close to the outer wall, and the fluctuation amplitude slightly increases.

Fig. 9 shows the azimuthal velocity and temperature fluctuations at a monitoring point of $R=1.5$ on the free surface when $B i=1.0$. Obviously, when Marangoni number is smaller, such as $M a=6000$, the velocity and the temperature at the monitoring point oscillate

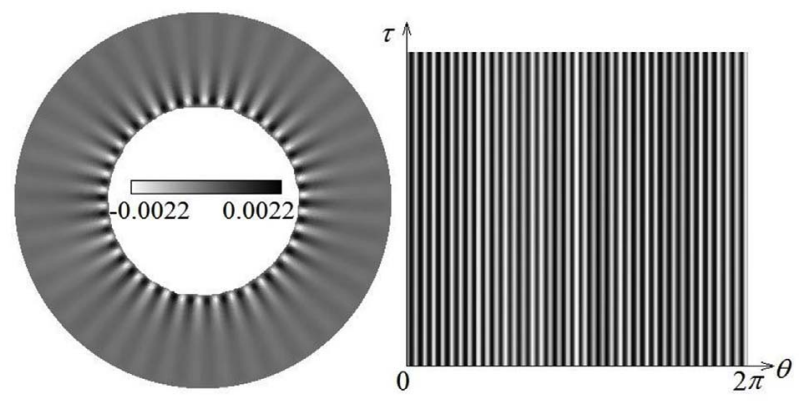

(a) $B i=0.125$

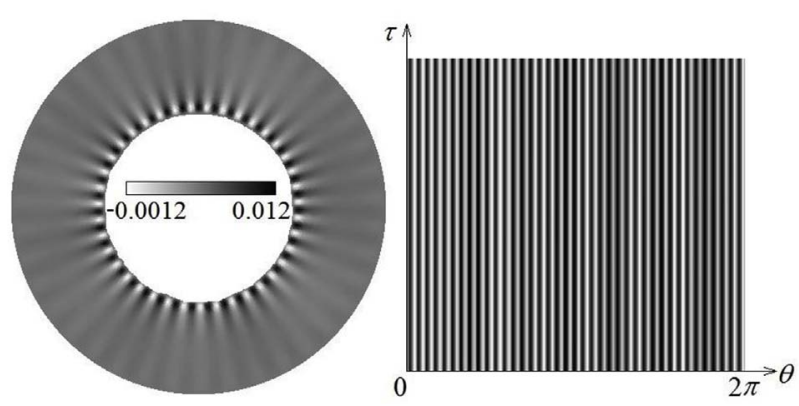

(b) $B i=0.25$

Fig. 4. Temperature fluctuation (left) and STD (right) at $R=1.16$ on free surface for 3DSF at $M a=4600$. 


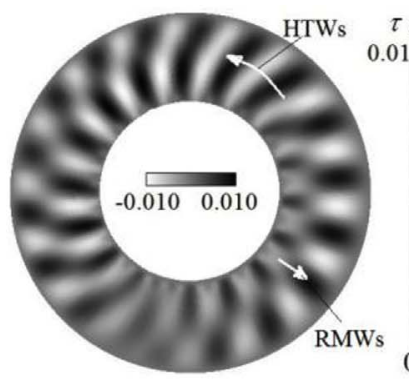

(a) $M a=5000$, RMWs + HTWs

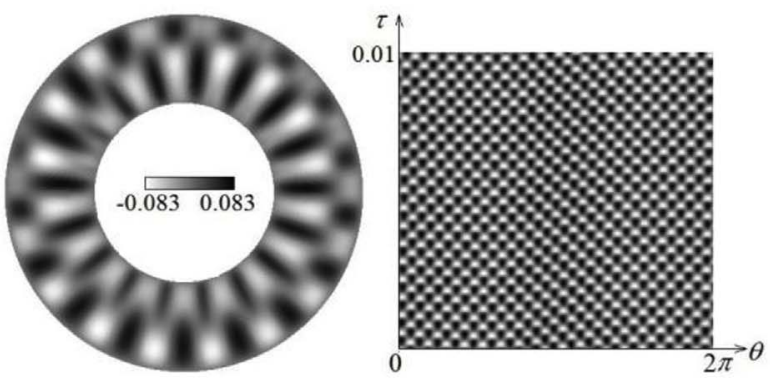

(c) $M a=8000$, RMWs

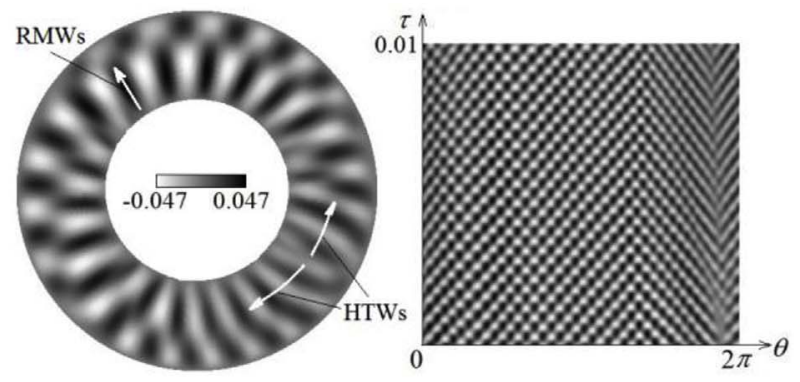

(b) $M a=6000$, RMWs+HTWs

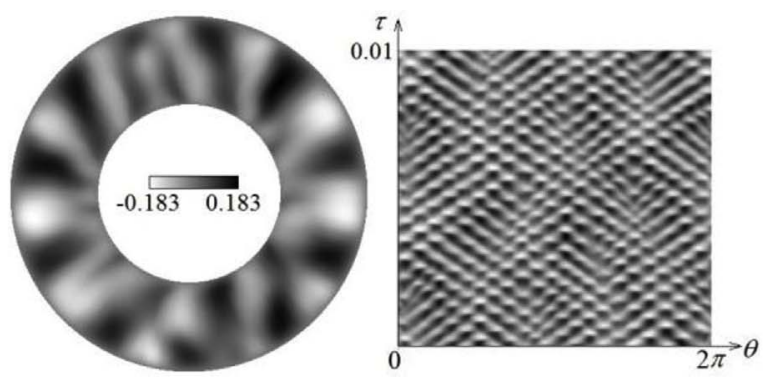

(d) $M a=12000, \mathrm{CF}$

Fig. 5. Snapshots of surface temperature fluctuation (left) and STD (right) at $R=1.5$ on free surface for 3-D oscillatory flow at $B i=0.25$.

periodically. When the HTWs propagate along a clockwise, the temperature fluctuation always lags behind the velocity fluctuation, and the phase difference is about $(6 / 5) \pi$, as shown in Fig. 9 (a). While the HTWs propagate along a counterclockwise, although the temperature fluctuation is also behind the velocity fluctuation, the phase difference between the two fluctuations is greatly reduced, only about $(1 / 10) \pi$, as shown in Fig. 9(b). It should be noted that the clockwise waves and the counterclockwise waves are equivalent in an axisymmetric annular pool without rotation. Near the critical point, the phase difference is definitely the same. However, with the increase of Marangoni number, the

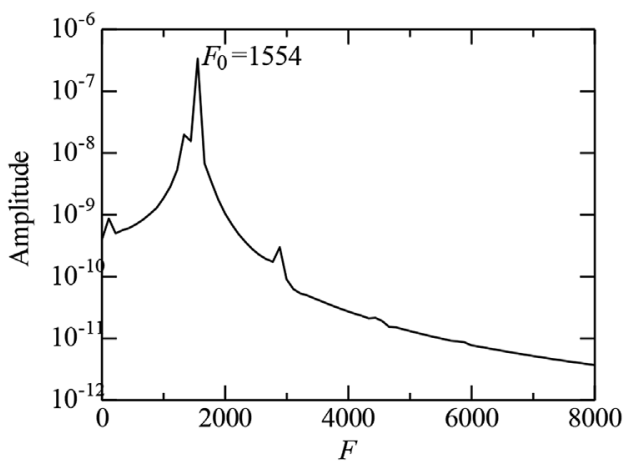

(a) $M a=5000$, RMWs + HTWs

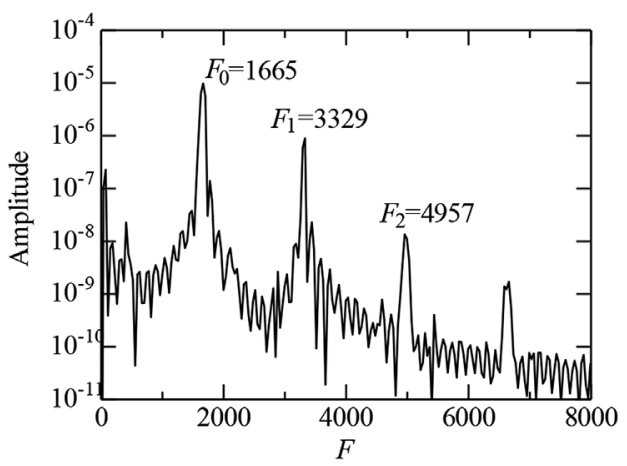

(c) $M a=8000$, RMWs

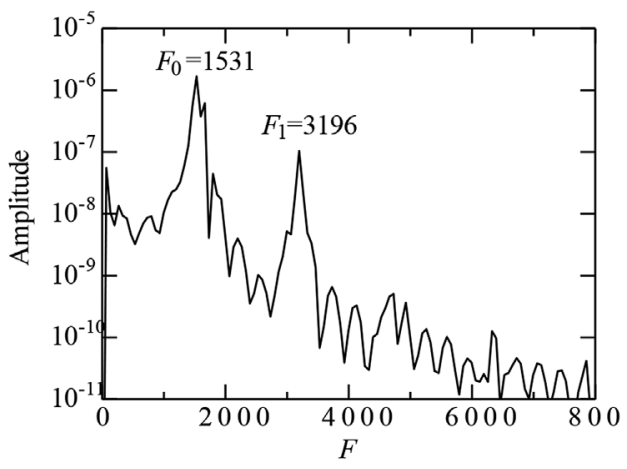

(b) $M a=6000$, RMWs + HTWs

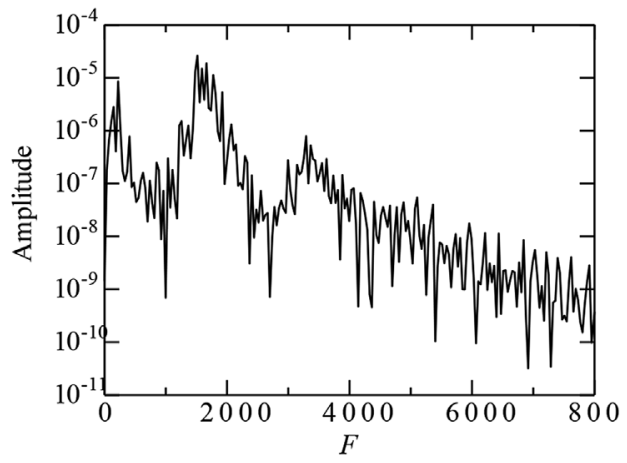

(d) $M a=12000, \mathrm{CF}$
Fig. 6. The frequency spectrums of radial velocity at $R=1.5$ of the free surface when $B i=0.25$. 


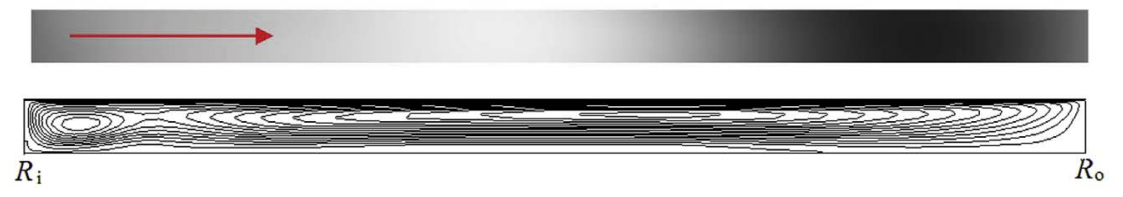

Fig. 7. Temperature fluctuation (up) and pseudo streamlines (down) during one period on a meridional plane at $M a=8000$. $\delta \psi=5$.

(a) $\tau=\tau_{0}, \psi_{\max }=91.91$

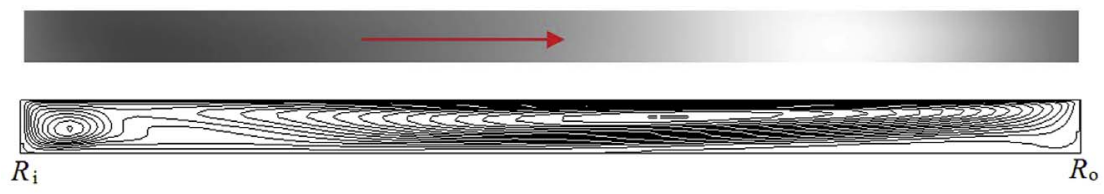

(b) $\tau=\tau_{0}+\tau_{\mathrm{p}} / 4, \psi_{\max }=108.23$

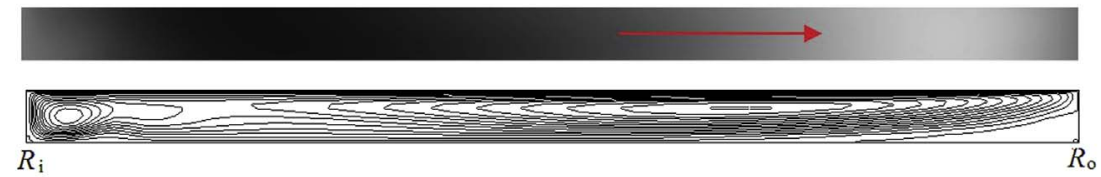

(c) $\tau=\tau_{0}+2 \tau_{\mathrm{p}} / 4, \psi_{\max }=90.46$

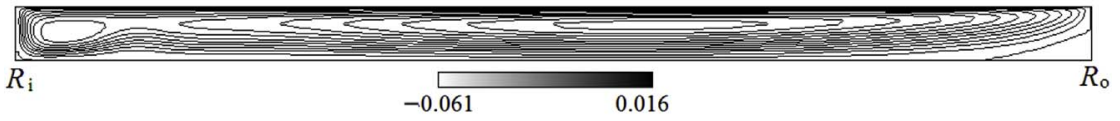

(d) $\tau=\tau_{0}+3 \tau_{\mathrm{p}} / 4, \psi_{\max }=81.14$

flow is enhanced and the phase difference also changes. For the HTWs propagating along different directions, although the phase difference between velocity and temperature fluctuations is different, the fluctuation period is the same, and the fundamental frequency is $F_{0}=1517$.

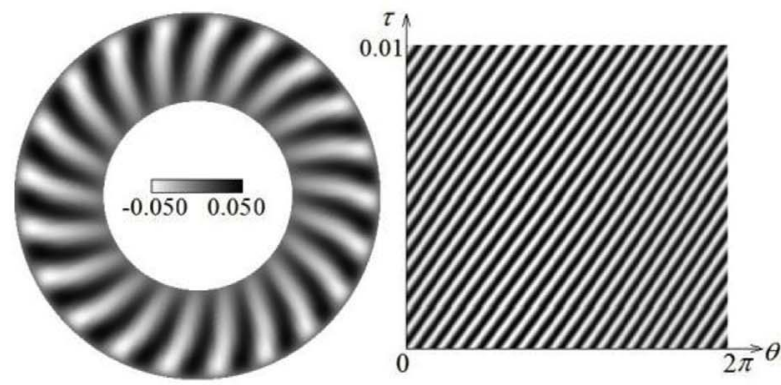

(a) $B i=0.5$, C.C.W.

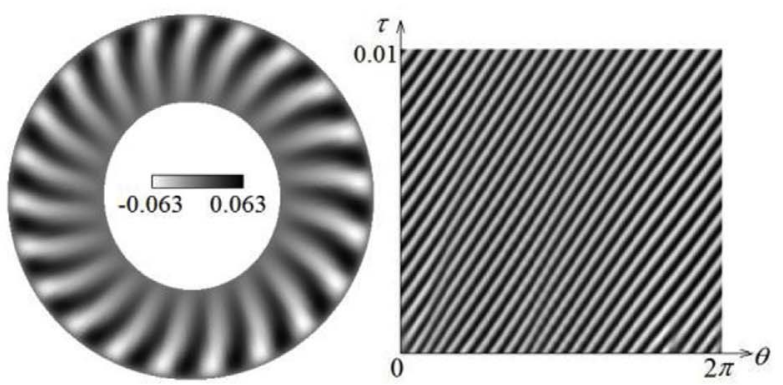

(c) $B i=0.75$, C.C.W.
As Marangoni number increases, the flow is strengthened. For the counterclockwise and clockwise propagating HTWs, Marangoni numbers of the flow pattern transition are not the same. For example, at $M a=8000$, when the flow and temperature fields for the clockwise propagating HTWs at $M a=6000$ are taken as the initial conditions, the

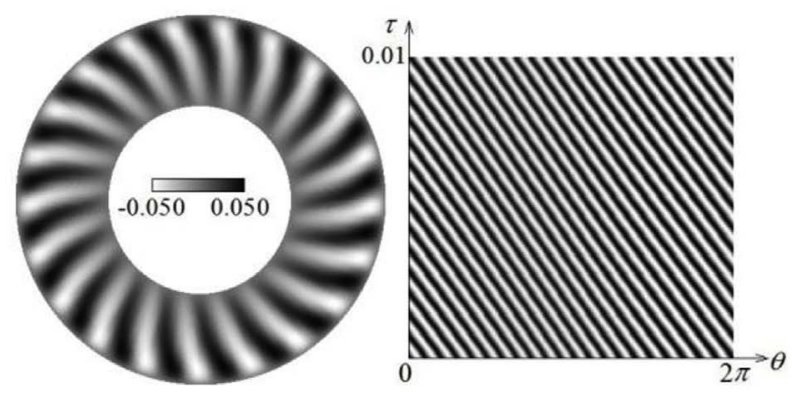

(b) $B i=0.5$, C.W.

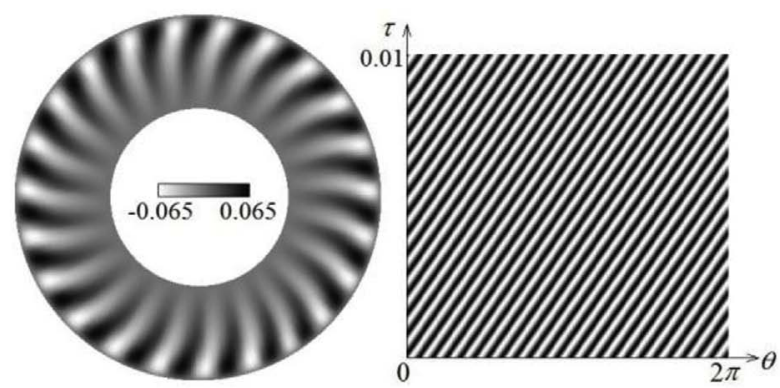

(d) $B i=1.0$, C.C.W

Fig. 8. Snapshots of surface temperature fluctuation (left) and STD (right) at $R=1.5$ on free surface for the HTWs at $M a=6000$. 


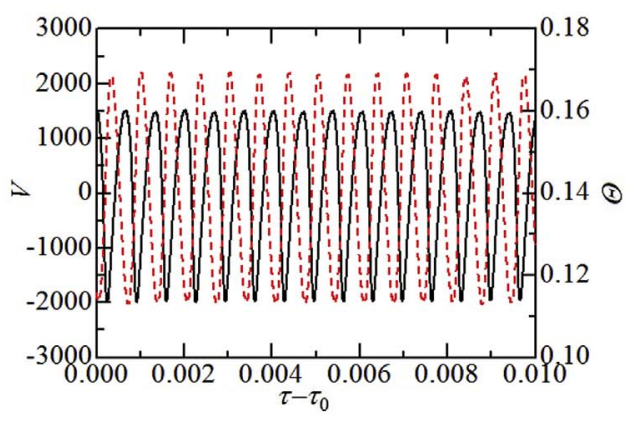

(a) $M a=6000$, HTWs, C.W.

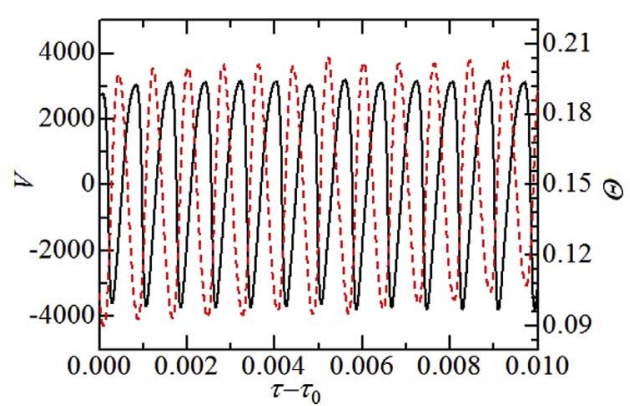

(c) $M a=8000$, HTWs, C.W.

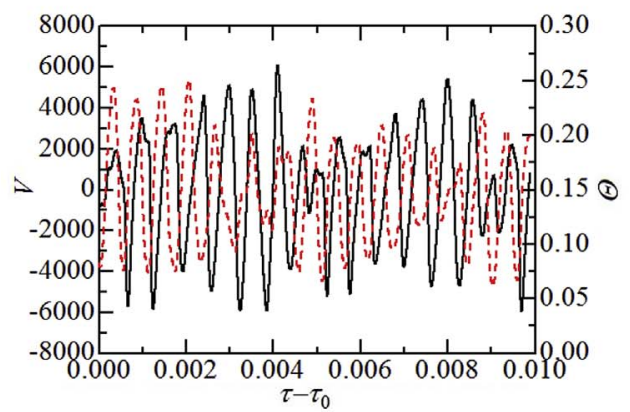

(e) $M a=10000, \mathrm{CF}$

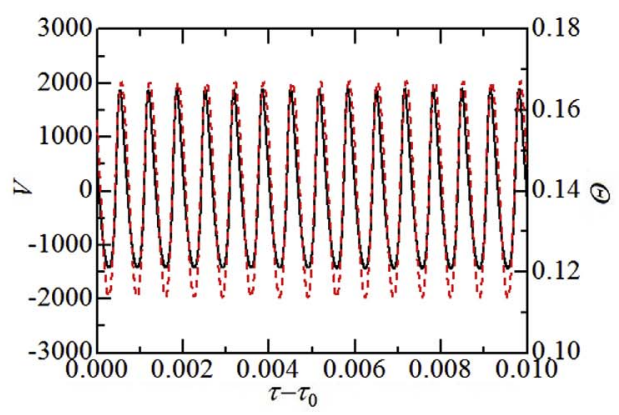

(b) $M a=6000$, HTWs, C.C.W.

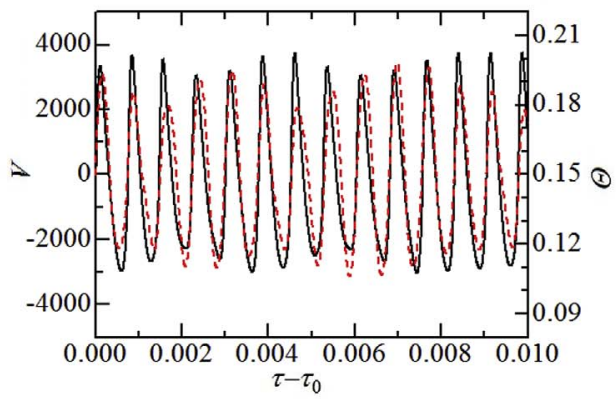

(d) $M a=8000$, HTWs, C.W.+C.C.W.

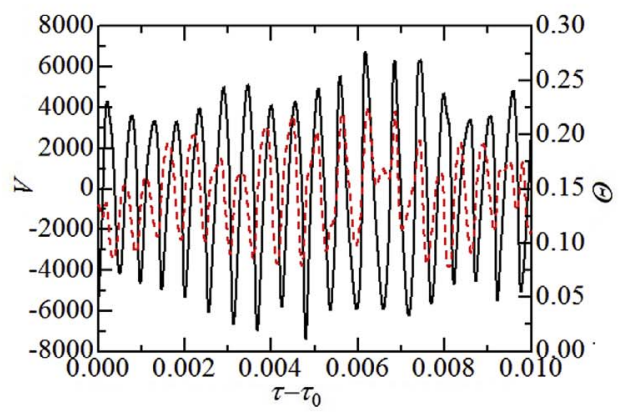

(f) $M a=12000, \mathrm{CF}$
Fig. 9. The azimuthal velocity (solid line) and temperature (dashed line) fluctuations at $R=1.5$ on the free surface when $B i=1.0$. wave pattern remains the same after a long time calculation. The snapshot of surface temperature fluctuation on the free surface is shown in Fig. 10(a). In this case, the temperature fluctuation at a monitoring point of $R=1.5$ still lag behind the velocity fluctuation, and the phase difference is also $(6 / 5) \pi$, as shown in Fig. 9 (c). Contrary, when $M a=8000$, the anticlockwise propagation HTWs at $M a=6000$ will evolve into a plurality of HTWs moving in different directions after a long period of calculation, and wave sources and wave sinks are basically fixed. Therefore, the STD consists of a plurality of parallel slant lines, as shown in Fig. 10 (b). The phase difference between the velocity and temperature fluctuations is varied, as shown in Fig. 9 (d), indicating that the fluctuations at this time are more complex. In addition, the fluctuations of velocity and temperature are mainly located at the outside of the liquid pool, which is different from that at $B i=0.25$. Many studies on thermocapillary convection under adiabatic free surface $[6,38]$ indicate that in the flow evolution, there exists the coexistence of many flow patterns under the same Marangoni number or thermocapillary Reynolds number. The present results show that when there exists surface heat dissipation, the coexistence of multiple flow patterns under the same Marangoni number is also observed.

When Marangoni number continues to increase, thermocapillary convection will be further enhanced, and the velocity and temperature fluctuations become more complex. The fluctuation process is no longer periodic, and the fluctuation amplitude is constantly changing, as shown in Fig. 9 (e) and (f). At the same time, the HTWs on the free surface gradually disappear, the spatial distribution of velocity and temperature fluctuations is no longer regular, the snapshots of surface temperature fluctuation and the STD at $R=1.5$ are shown in Fig. 10 (c) and (d).

Fig. 11 shows the frequency spectrum of azimuthal velocity fluctuation at a monitoring point of $R=1.5$ during the flow pattern evolution when $B i=1.0$. At $M a=8000$, the flow pattern is the HTWs, so there is always a dominant fluctuation frequency, as shown in Fig. 11 (a) and (b). In this case, Marangoni number is larger and the flow is stronger, which leads to the complicated velocity and temperature fluctuations. Many corresponding harmonic frequencies appear. For example, for a single group HTWs along the clockwise rotation, the fundamental fluctuation frequency is $F_{0}=1258$ and the harmonic frequency is $F_{1}=2441 \approx 2 F_{0}$. For multiple groups of HTWs along the two direction rotation, the fundamental fluctuation frequency is $F_{0}=1332$, the harmonic frequencies are $F_{1}=2663 \approx 2 F_{0}$ and $F_{2}=3995 \approx(3 / 2) F_{1} \sim 3 F_{0}$. Therefore, the harmonic frequency always appears with the multiple of the fundamental fluctuation frequency. When Marangoni number exceeds 9000, the periodicity of temperature and velocity fluctuations gradually disappears. On the spectrum diagram, the noise increases. It is difficult to determine an obvious fluctuation frequency, indicating that the flow at this moment has evolved into the chaotic flow, as shown in Fig. 11 (c) and (d). Therefore, when 

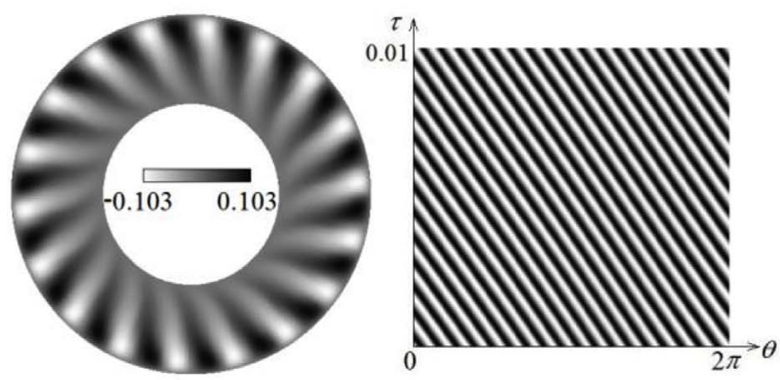

(a) $M a=8000$, HTWs, C.W.

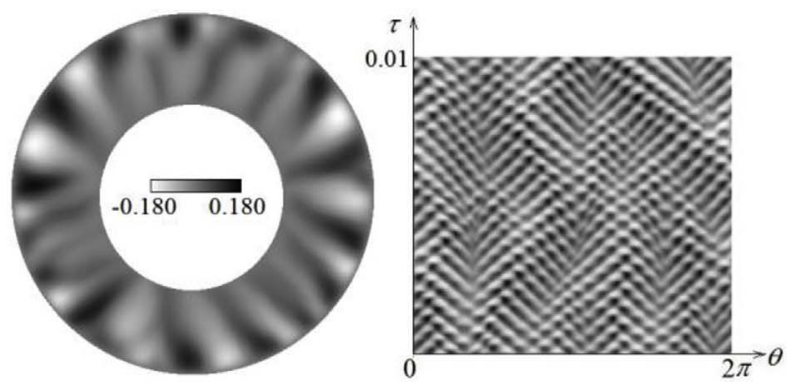

(c) $M a=10000, \mathrm{CF}$

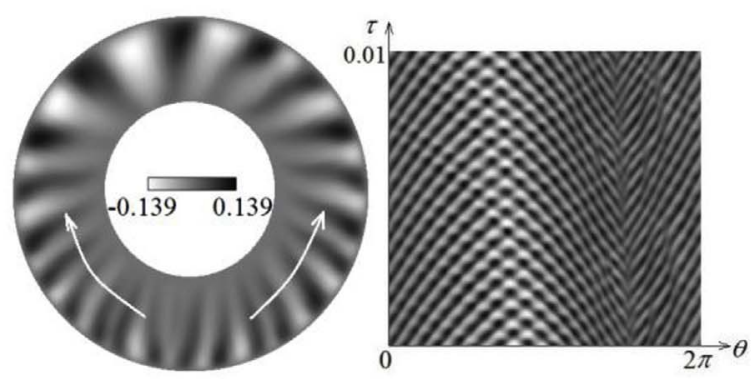

(b) $M a=8000$, HTWs, C.W.+C.C.W.

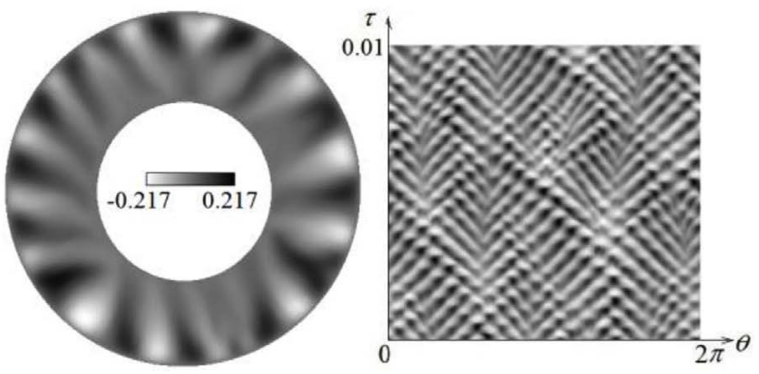

(d) $M a=12000, \mathrm{CF}$

Fig. 10. Snapshots of surface temperature fluctuation (left) on the free surface and STD (right) at $R=1.5$ when $B i=1.0$.

Biot number is larger, the HTWs are also gradually evolved into the chaotic state by the period-halving bifurcation.

\subsection{Flow pattern transition diagram}

The above analysis results show that when surface heat dissipation

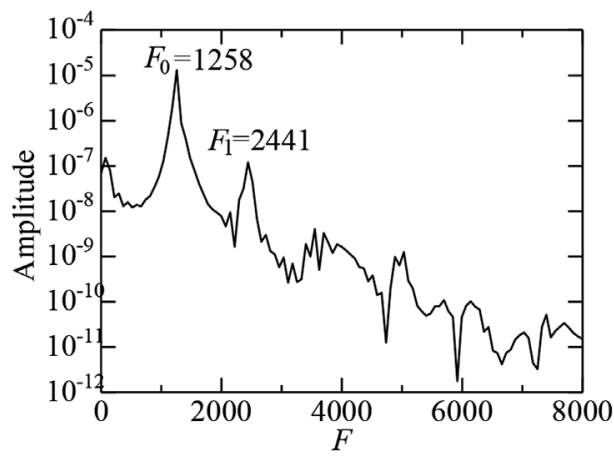

(a) $M a=8000$, HTWs, C.W.

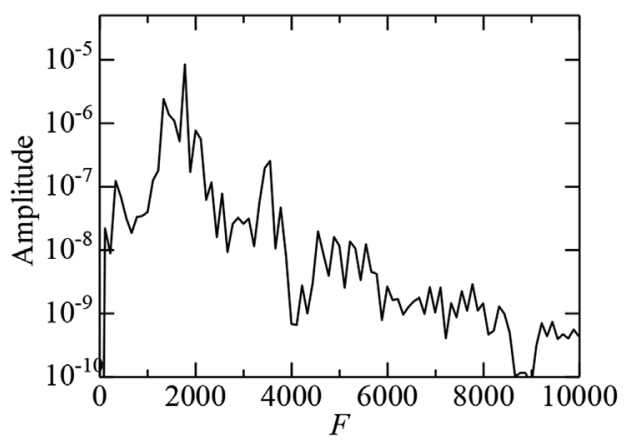

(c) $M a=10000, \mathrm{CF}$

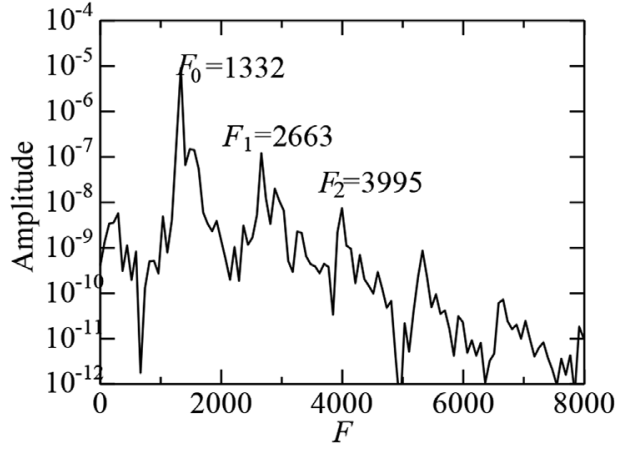

(b) $M a=8000$, HTWs, C.W.+C.C.W.

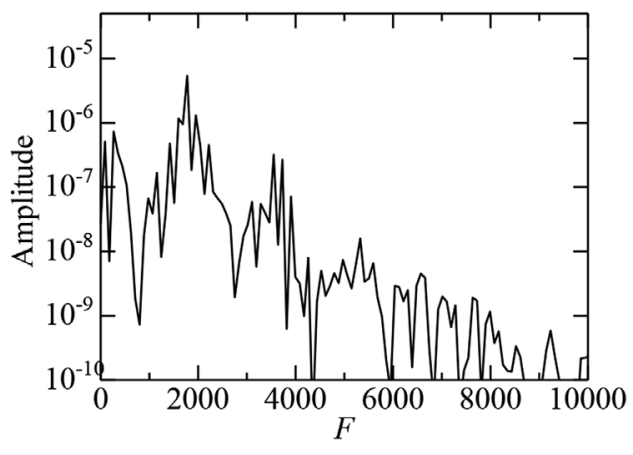

(b) $M a=12000, \mathrm{CF}$
Fig. 11. Frequency spectrum of azimuthal velocity fluctuation at a monitoring point of $R=1.5$ when $B i=1.0$. 


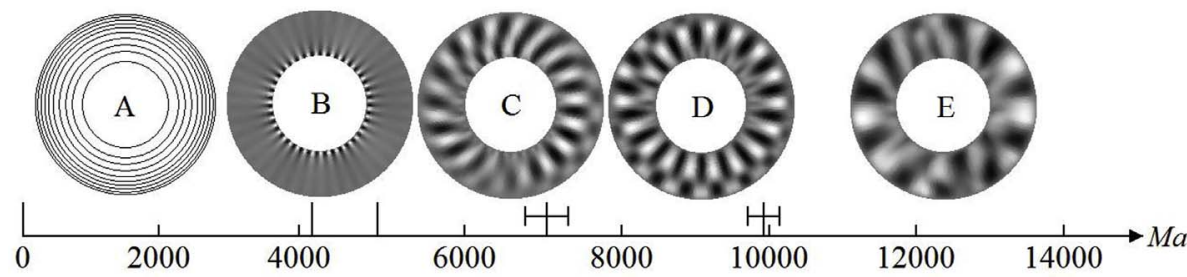

(a) $B i=0.25$

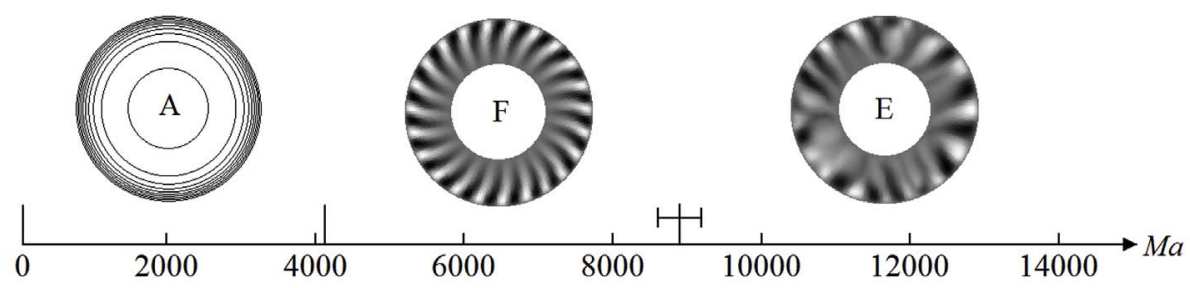

(b) $B i=1.0$

flow pattern transition at different Biot numbers. Obviously, the flow pattern is more abundant and its evolution is more complicated at a small Biot number. But in this case, the pure HTW is not observed in the liquid pool. Meanwhile, the ranges of Marangoni number and Biot number for the 3-D steady state flow existence are narrow. For example, when $B i=0.25$, the 3-D steady flow exists only in the range of $4215 \leq M a<4800$. On the other hand, during the various 3-D unsteady flow pattern transition, Marangoni number is not unique, but there is a transition Marangoni number interval, which means that there may be different 3-D unsteady flow patterns under the same Marangoni number $[6,39]$. When Biot number is larger, the flow pattern transition becomes simpler. In this case, 2-D steady axisymmetric flow bifurcates directly to the HTWs after the flow destabilization, and the Marangoni range of the HTWs existence is wide.

Fig. 13 shows the variations of the maximum temperature fluctuation amplitude $A_{\max }\left(=\Theta_{\max }-\Theta_{\min }\right)$ on the free surface, the wave number $m$ and the fundamental frequency $F_{0}$ with Marangoni number. It can be seen that with the increase of Marangoni number, the flow is enhanced, and the temperature fluctuation amplitude on the free surface increases gradually. When Marangoni number is smaller, surface heat dissipation will make the temperature gradient near the outer wall increase, and the flow is strengthened, so the temperature fluctuation amplitude becomes larger. Conversely, when Marangoni number is larger, the flow is confined to the outer wall due to surface heat dissipation. Compared with adiabatic free surface, the flow is weakened so that the maximum temperature fluctuation amplitude decreases. At the same time, when the free surface is adiabatic, the maximum temperature fluctuation on the free surface is always located near the inner wall. With the increase of surface heat dissipation, the radial position of the maximum temperature fluctuation on the free surface will gradually move towards the outer wall.

When the flow is 3-D steady flow, the wave number is large and varies little. When 3-D steady flow evolves into 3-D unsteady flow, the wave number will reduce suddenly. Then, with the increase of Marangoni number, for the combination of HTWs and RMWs, the wave number will increase slightly. When the flow pattern becomes the RMW, the wave number will decrease until the flow transits to chaotic state. When $B i=1.0$, in a wide range of Marangoni numbers, the wave number of HTWs is basically constant. However, at a larger Marangoni number, the wave number decreases gradually with Marangoni number. For 3-D oscillatory flow, the fundamental fluctuation frequency increases with Marangoni number at a small Biot number, but decreases at a large Biot number.
Fig. 12. Flow pattern transition process. A: 2DSF; B: 3DSF; C: HTWs + RMWs; D: RMWs; E: CF; F: HTWs.

\section{Conclusions}

A series of 3-D numerical simulations are performed to analyze clearly the flow bifurcation routes to chaos of thermocapillary convection with surface heat dissipation for low Prandtl number fluid in shallow annular pool. The following conclusions were obtained.

(1) Surface heat dissipation has a great influence on the flow pattern bifurcation processes and routes. The flow pattern bifurcation route at a large Biot number is simpler than that at a small Biot number.

(2) When surface heat dissipation is smaller, the flow bifurcation sequence of thermocapillary convection is 2-D axisymmetric steady flow $\rightarrow$ 3-D steady flow $\rightarrow$ coexisting hydrothermal waves and radial moving waves $\rightarrow$ radial moving waves $\rightarrow$ chaos with the

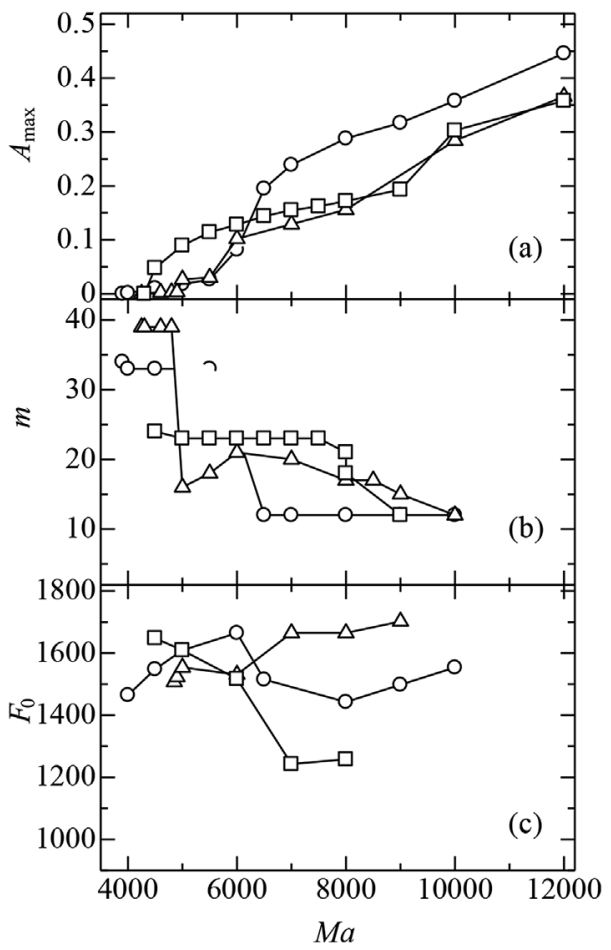

Fig. 13. Variations of the maximum dimensionless temperature amplitude $A_{\max }$ (a), wave number $m$ (b), and fundamental frequency $F_{0}$ (c) with Marangoni number. When there coexist multiple flow patterns under the same Marangoni number, values in the figure denote these after flow pattern transition. $\bigcirc: B i=0 ; \triangle: B i=0.25 ; \square: B i=1.0$. 
increase of Marangoni number. When surface heat dissipation is larger, the bifurcation sequence is 2-D axisymmetric steady flow $\rightarrow$ hydrothermal wave $\rightarrow$ chaos.

(3) The temperature fluctuation amplitude on the free surface increases gradually with the increase of Marangoni number. However, the wave number and the fluctuation frequency depend mainly on the flow pattern and Marangoni number. Every bifurcation of thermocapillary convection is always accompanied by a sudden variation of the wave number. The fundamental fluctuation frequency increases with Marangoni number at a small Biot number, but decreases at a large Biot number.

\section{Acknowledgement}

This work is supported by National Natural Science Foundation of China (Grant No. 51776022, 11532015).

\section{References}

[1] W.R. Hu, Z.M. Tang, K. Li, Thermocapillary convection in floating zones, Appl Mech Rev 61 (1) (2008) 010803.

[2] A.A. Avramenko, I.V. Shevchuk, S. Harmand, A.I. Tyrinov, Thermocapillary instability in an evaporating two-dimensional thin layer film, Int J Heat Mass Transf 91 (2015) 77-88.

[3] S.P. Aktershev, Stability of the heated liquid film in the presence of the thermocapillary effect, Thermophys Aeromechanics 20 (1) (2013) 1-16.

[4] B. Sobac, D. Brutin, Thermocapillary instabilities in an evaporating drop deposited onto a heated substrate, Phys Fluids 24 (3) (2012) 032103.

[5] J.P. Gollub, S.V. Benson, Many routs to turbulent convection, J Fluid Mech 100 (3) (1980) 449-470.

[6] M.K. Smith, S.H. Davis, Instabilities of dynamic thermocapillary liquid layers. 1. Convective instabilities, J Fluid Mech 132 (7) (1983) 119-144.

[7] M.K. Smith, S.H. Davis, Instabilities of dynamic thermocapillary liquid layers. 2. Surface-wave instabilities, J Fluid Mech 132 (7) (1983) 145-162.

[8] M.K. Smith, Instability mechanisms in dynamic thermocapillary liquid layers, Phys Fluids 29 (10) (1986) 3182-3186.

[9] D. Villers, J.K. Platten, Coupled buoyancy and Marangoni convection in acetone: experiments and comparison with numerical simulations, J Fluid Mech 234 (-1) (1991) 487-510.

[10] E. Bucchignani, D. Mansutti, Horizontal thermal convection in a shallow cavity: oscillatory regimes and transition to chaos, Int J Numer Methods Heat Fluid Flow 10 (2000) 179-195.

[11] P. Zhu, L. Duan, Q. Kang, Transition to chaos in thermocapillary convection, Int J Heat Mass Transf 57 (2013) 457-464.

[12] Y.R. Li, N. Imaishi, T. Azami, T. Hibiya, Three-dimensional oscillatory flow in a thin annular pool of silicon melt, J Cryst Growth 260 (2004) 28-42.

[13] Y.R. Li, L. Peng, Y. Akiyama, N. Imaishi, Three-dimensional numerical simulation of thermocapillary flow of moderate Prandtl number fluid in annular pool, J Cryst Growth 259 (2003) 374-387.

[14] J.C. Chen, Y.R. Li, J.J. Yu, L. Zhang, C.M. Wu, Flow pattern transition of thermalsolutal capillary convection with the capillary ratio of -1 in a shallow annular pool, Int J Heat Mass Transf 95 (2016) 1-6.

[15] J.J. Yu, Y.R. Li, C.M. Wu, J.C. Chen, Three-dimensional thermocapillary-buoyancy flow of a binary mixture with Soret effect in a shallow annular pool, Int J Heat Mass Transf 90 (2015) 1071-1081.
[16] P. Gillon, G.M. Homsy, Combined thermocapillary-buoyancy convection in a cavity: an experimental study, Phys Fluids 8 (11) (1996) 2953-2963.

[17] J.J. Yu, D.F. Ruan, Y.R. Li, J.C. Chen, Experimental study on thermocapillary convection of binary mixture in a shallow annular pool with radial temperature gradient, Exp Therm Fluid Sci 61 (2015) 79-86.

[18] J. Burguete, N. Mukolobwiez, F. Daviaud, N. Garnier, A. Chiffaudel, Buoyantthermocapillary instabilities in extended liquid layers subjected to a horizontal temperature gradient, Phys Fluids 13 (2001) 2773-2787.

[19] S. Benz, D. Schwabe, The three-dimensional stationary instability in dynamic thermocapillary shallow cavities, Exp Fluids 31 (2001) 409-416.

[20] F. Daviaud, J.M. Vince, Traveling waves in a fluid layer subjected to a horizontal temperature gradient, Phys Rev E 48 (1993) 4432-4436.

[21] N. Garnier, A. Chiffaudel, Two dimensional hydrothermal waves in an extend cylindrical vessel, Eur Phys J B 19 (2001) 87-95.

[22] C.J. Jing, N. Imaishi, S. Yasuhiro, Y. Miyazawa, Three-dimensional numerical simulation of spoke pattern in oxide melt, J Cryst Growth 200 (1999) 204-212.

[23] N. Zhang, D.F. Chao, Mechanisms of convection instability in thin liquid layers induced by evaporation, Int Commun Heat Mass Transf 26 (1999) 1069-1080.

[24] Y. Li, R. Grigoriev, M. Yoda, Experimental study of the effect of noncondensables on buoyancy-thermocapillary convection in a volatile low-viscosity silicone oil, Phys Fluids 26 (12) (2014) 122112.

[25] Z.Q. Zhu, Q.S. Liu, Coupling of thermocapillary convection and evaporation effect in a liquid layer when the evaporating interface is open to air, Chin Sci Bull 55 (2010) 233-238.

[26] Z.Q. Zhu, Q.S. Liu, Experimental investigation of thermocapillary convection in a liquid layer with evaporating interface, Chin Phys Lett 5 (2008) 4046-4049.

[27] J. Yan, Q.S. Liu, R. Liu, Coupling of evaporation and thermocapillary convection in a liquid layer with mas and heat exchanging interface, Chin Phys Lett 25 (2008) 608-611.

[28] S. Hoyas, P. Fajardo, A. Gil, M.J. Perez-Quiles, Analysis of bifurcations in a BénardMarangoni problem: gravitational effects, Int J Heat Mass Transf 73 (2014) 33-41.

[29] S. Hoyas, P. Fajardo, A. Gil, M.J. Perez-Quiles, Influence of geometrical parameters on the linear stability of a Bénard-Marangoni problem, Phys Rev E 93 (2016) 043105.

[30] A.J. Torregrosa, S. Hoyas, M.J. Perez-Quiles, J.M. Mompo-Laborda, Bifurcation diversity in an annular pool heated from below: Prandtl and Biot numbers effects, Commun Comput Phys 13 (2013) 428-441.

[31] F. Doumenc, T. Boeck, B. Guerrier, M. Rossi, Transit Rayleigh-Bénard-Marangoni convection due to evaporation: a linear non-normal stability analysis, J Fluid Mech 648 (2010) 521-539.

[32] O. Touazi, E. Chénier, F. Doumenc, B. Guerrier, Simulation of transient RayleighBénard-Marangoni convection induced by evaporation, Int J Heat Mass Transf 53 (2010) 656-664.

[33] T. Azami, S. Nakamura, M. Eguchi, T. Hibiya, The role of surface-tension-driven flow in the formation of a surface pattern on a Czochralski silicon melt, J Cryst Growth 233 (2001) 99-107.

[34] Q.S. Liu, Y. Wang, Y. Ji, Coupling mechanism of evaporation phase-change and interfacial flow, J Eng Thermophys 31 (2010) 1751-1754 (In Chinese).

[35] L. Zhang, Y.R. Li, C.M. Wu, Effect of surface evaporation on steady thermocapillary convection in an annular pool, Microgravity Sci Technol 28 (2016) 499-509.

[36] L. Zhang, Y.R. Li, C.M. Wu, Effect of surface heat dissipation on thermocapillary convection of low Prandtl number fluid in a shallow annular pool, Int J Heat Mass Transf 110 (2017) 660-667.

[37] L. Peng, Y.R. Li, W.Y. Shi, N. Imaishi, Three-dimensional thermocapillary-buoyancy flow of silicone oil in a differentially heated annular pool, Int J Heat Mass Transf 50 (2007) 872-880.

[38] Y.R. Li, L. Peng, S.Y. Wu, Bifurcation of thermocapillary convection in a shallow annular pool of silicon, Melt. Acta Mech Sin 23 (1) (2007) 43-48.

[39] W.Y. Shi, Y.R. Li, L. Peng, D.L. Zeng, N. Imaishi, Natural characteristics of hydrothermal wave in a shallow annular pool, Chin J Comput Mech 26 (1) (2009) 59-65. 